\title{
Erratum to: Evidence-based indications for hindfoot endoscopy
}

\author{
Pietro Spennacchio $^{1} \cdot$ Davide Cucchi $^{1,2} \cdot$ Pietro S. Randelli $^{1,2} \cdot$ Niek C. van Dijk $^{3}$
}

Published online: 12 March 2016

(C) European Society of Sports Traumatology, Knee Surgery, Arthroscopy (ESSKA) 2016

\section{Erratum to: Knee Surg Sports Traumatol Arthrosc DOI 10.1007/s00167-015-3965-1}

The author would like to correct the following errors in the online publication of the article:

In the "Hindfoot periarticular area" section, under the sub-heading "Posterior ankle impingement", the seventh and eighth paragraph should read as:

The AOFAS score was the most frequently used clinical outcome score; where used, cumulative results showed average post-operative score of 90.6 points $[1,2,11,22$, $24,27,32,42,50,56,64,68]$ and pre- to post-operative increase by an average of 24.0 points [1, 2, 24, 27, 32, 42, $50,56,64]$.

Six studies reported results of isolated os trigonum excision in 99 ankles [2, 18, 22, 24, 33, 46]: the pre- to postoperative increase in AOFAS was available for 22 patients and reached 33.7 points $[2,24]$, while pre- to post-operative VAS decrease was available for 62 patients and was $>6$ points [18, 24, 33].

The online version of the original article can be found under doi:10.1007/s00167-015-3965-1.

Davide Cucchi

d.cucchi@gmail.com

1 IRCCS Policlinico San Donato, Via Morandi 30, 20097 San

Donato Milanese, Milan, Italy

2 Dipartimento di Scienze Biomediche per la Salute, Università degli Studi di Milano, Via Mangiagalli 31, 20133 Milan, Italy

3 Department of Orthopaedic Surgery, Academic Medical Center, University of Amsterdam, Meibergdreef 9, 1105 AZ Amsterdam Zuid-Oost, The Netherlands
In the "Articular joint spaces" section, under the subheading "Subtalar joint articular space", the first paragraph should read as follows:

Osteoarthritis Level of evidence IV and V Degenerative changes of the subtalar joint are reported in the literature as indications for hindfoot endoscopy in prone position, eitherby means of talocalcaneal arthrodesis $[3-5,10,29$, $30,38,39,57]$ or arthroscopic osteophyte resection and debridement [39, 42]. Seven retrospective case series adequately documented the clinical results of 73 procedures of posterior arthroscopic subtalar arthrodesis (PASTA) [3, 4, $10,29,30,38,57]$. Over these studies, post-traumatic or primary osteoarthritis were the preoperative indications for PASTA in more than $90 \%$ of the cases. In five cases, subtalar arthropathy was related to a tarsal coalition [3, 4]. Five of the seven series evaluated post-operative clinical outcome with the AOFAS score: the cumulative results showed average post-operative score by 80.3 points and pre- to postoperative increase by an average of 40.4 points $[3,4,29,38,57]$

The original article has been updated accordingly. 